\title{
Stratégies de prospect du droit solaire par l'immersion
}

\author{
Laila Koubaa Turki ${ }^{1}{ }^{[1]}$, Khaoula Raboudi $^{1}$, et Abdelkader Ben Saci ${ }^{2}$ \\ ${ }^{1}$ Univ. Carthage, ENAU, Equipe de recherche M2A, Sidi Bou Saï, Tunisie \\ ${ }^{2}$ Univ. Grenoble Alpes, CNRS, ENSAG, AAU-CRESSON, 38000 Grenoble, France
}

\begin{abstract}
Résumé. Cet article propose des stratégies du droit solaire basées sur l'optimisation du temps de coupure d'ombrage et des clôtures d'ombres. Il vise à minimiser l'empreinte carbone des bâtiments en exploitant au mieux les ressources utiles du contexte, notamment le parcellaire. Il propose la notion du retrait propre pour restreindre l'ombre à l'intérieur de la parcelle pendant le temps de coupure d'ombrage. Le retrait propre mutualisé par la négociation entre voisins optimise le potentiel solaire et la densité urbaine. Il sert à établir une réglementation urbaine dynamique qui se base sur l'immersion via une maquette numérique paramétrique. Celle-ci permet de définir des nouvelles stratégies de droit solaire favorisant à la fois la densification urbaine et une meilleure exploitation des ressources utiles de l'environnement.
\end{abstract}

Mots-clés. Droit solaire par l'immersion, modélisation paramétrique, prospect, réglementation morphologique urbaine, stratégies, négociation.

\begin{abstract}
This article proposes strategies of solar right based on the optimization of the shading cut-off time and shadow fences. It aims to minimize the carbon footprint of buildings by making the most of the useful resources of the context, particularly the plot. It proposes the concept of proper withdrawal to restrict the shade within the parcel during a shade cut-off time. The proper withdrawal mutualized by negotiation between neighbors optimizes solar potential and density. It is used to establish a dynamic urban regulation based on immersion via a parametric numerical model. This allows to define new strategies of solar right that promote both urban densification and a better exploitation of the useful resources of the environment.
\end{abstract}

Keywords. Solar right by immersion, parametric modeling, prospect, urban morphological regulation, strategies, negotiation.

\section{Introduction}

La règle du prospect de la limite mitoyenne définit l'implantation des constructions par rapport aux limites séparatives des parcelles. Elle détermine la distance minimale d'une

\footnotetext{
${ }^{[1]}$ Corresponding author : koubaa.leila@yahoo.fr
} 
construction par rapport à la limite mitoyenne en fonction de sa hauteur. L'objectif de cette règle est de garantir l'accès à l'ensoleillement et une bonne aération (Belkaid, 2014). L'accès à l'ensoleillement est une préoccupation ancienne qu'on trouve dans plusieurs cultures. Dans l'architecture vernaculaire maghrébine, une attention particulière était portée à l'ombrage et l'ensoleillement. Celui-ci fût l'objet d'intérêt des hygiénistes (Harzallah, 2007) et considéré comme facteur de santé de l'architecture du XXème siècle (Hobday et Dancer, 2013). L'accès à l'ensoleillement est de plus en plus formalisé et pris en charge par des modèles et des outils informatiques (Siret, 1997). La modélisation paramétrique et la maquette numérique facilitent actuellement la génération de morphologie urbaine sous contrainte d'ensoleillement à l'échelle du quartier (Vartholomaios, 2015) et de l'ilot (De Luca, 2017). La détermination de règles urbaines d'ensoleillement qui tiennent compte des attentes des habitants nécessite la recherche de nouveaux dispositifs numériques dynamiques et interactifs.

En Tunisie, le prospect par rapport à la limite mitoyenne est calculé selon un rapport de proportion en fonction de la hauteur de façade. Le rapport recommandé par la réglementation urbaine en vigueur est de H/2 (P.A.C.T, 2016). La règle de prospect, telle que formulée actuellement, limite l'accès à l'ensoleillement en hiver, entraine des problèmes de surchauffe en été et freine la densification urbaine alors qu'il y a un besoin grandissant de terrains dans les agglomérations (Raboudi et al., 2011 ; Chabbi, 2004). Nous nous demandons alors s'il est possible de proposer, à l'aide des techniques numériques actuelles, des stratégies de réglementation de prospect assurant un accès solaire optimal à plusieurs bâtisses implantées sur des parcelles voisines ?

Nous proposons de répondre à cette question à l'aide du concept de l'enveloppe solaire. En effet, ce dernier vise à préserver le droit solaire ${ }^{2}$ en milieu urbain. Il contraint l'enveloppe constructible de manière à assurer l'accès direct du soleil des bâtiments voisins pendant des périodes remarquables d'ensoleillement (Knowles, 1981). L'enveloppe solaire est définie par les données de la clôture d'ombre et du temps de coupure. Ce dernier désigné d'ombrage par Raboudi et Ben Saci (2014) est la période de temps d'accès solaire des bâtiments voisins. Il correspond à des périodes d'irradiation solaire maximale en hiver. La clôture d'ombre est une limite restrictive de projection d'ombre. Elle est définie actuellement aux pieds des constructions voisines en considérant un contexte urbain « statique » parce que prédéfini par le prospect réglementaire.

Cet article vise à améliorer la détermination des contraintes du temps de coupure d'ombrage et des clôtures d'ombre. Nous proposons de définir le temps de coupure d'ombrage par une étude de l'irradiation solaire avec la loi de Pareto ${ }^{3}$. Nous proposons cette loi pour définir le seuil des heures les plus pertinentes du point de vue du captage de l'irradiation solaire directe. Nous proposons également la notion de retrait propre pour délimiter la clôture d'ombre. Cette notion permet de considérer l'environnement urbain dynamique et d'envisager des modalités de mutualisation. Elle offre une nouvelle approche de morphologie qui intègre le potentiel d'ensoleillement. Elle permet de formaliser des stratégies de réglementation urbaine de prospect en vue d'une meilleure gouvernance de l'espace urbain à l'ère de la ville intelligente. Elle offre un instrument de décision pour l'établissement de règles urbaines respectant le droit solaire et impliquant les habitants.

\footnotetext{
${ }^{2}$ Le droit solaire définit les conditions d'accès au soleil dans le bâtiment. Il désigne l'aptitude d'une construction à recevoir la lumière du soleil dans les limites de la propriété, sans obstruction de la propriété voisine (Knowles, 1981).
}

${ }^{3}$ La loi de Pareto décrit un phénomène empirique constaté dans certains domaines : environ $80 \%$ des effets sont le produit de $20 \%$ des causes (Pareto, 1965). 


\section{Détermination des contraintes de l'enveloppe solaire}

\subsection{Détermination du temps de coupure d'ombrage}

Pour définir le temps de coupure d'ombrage, noté $\mathrm{T}_{\mathrm{o}}$, nous calculons l'irradiation solaire directe, notée $\mathrm{Rd}$, reçue par heure pour différentes orientations de façades au solstice d'hiver. Nous nous intéressons dans cet article à l'étude de l'éclairement solaire directe hivernal sans tenir compte des apports solaires diffus et des inter-réflexions. L'objectif est de définir les heures de captage optimal. Le calcul est effectué sur un mètre carré de façade avec le plugin Ladybug ${ }^{4}$.

Nous présentons dans ce qui suit le cas d'étude d'une parcelle d'habitat isolé située à la Goulette de Tunis à la station GPS 147017 (latitude 36.78, longitude 10.25). Nous calculons l'irradiation solaire directe de $8 \mathrm{~h}$ à $16 \mathrm{~h}$ pour différentes orientations de façades avec un pas de rotation des orientations des façades de $22.5^{\circ}$. La position $0^{\circ}$ indique la façade Nord et la position $180^{\circ}$ indique la façade Sud. Nous calculons également la somme des irradiations et le taux d'irradiation par heure (Tableau 1).

Tableau 1. Irradiation directe $\mathrm{Rd}\left(\mathrm{en} \mathrm{W} / \mathrm{m}^{2}\right.$ ) calculée le 21 décembre pour différentes orientations de façades avec un pas de $22.5^{\circ}$ à la station GPS 147017 (Goulette de Tunis : lat 36.78, long10.25).

\begin{tabular}{|c|c|c|c|c|c|c|c|c|c|}
\hline $\begin{array}{l}\text { Heure } \\
\text { Façade }\end{array}$ & $8 \mathrm{~h}$ & $9 \mathrm{~h}$ & $10 \mathrm{~h}$ & $11 \mathrm{~h}$ & $12 \mathrm{~h}$ & $13 \mathrm{~h}$ & $14 \mathrm{~h}$ & $15 \mathrm{~h}$ & $16 \mathrm{~h}$ \\
\hline $0^{\circ}$ Nord & 0,00 & 0,00 & 0,00 & 0,00 & 0,00 & 0,00 & 0,00 & 0,00 & 0,00 \\
\hline $22,5^{\circ}$ & 0,00 & 0,00 & 0,00 & 0,00 & 0,00 & 0,00 & 0,00 & 0,00 & 0,00 \\
\hline $45^{\circ}$ & 0,97 & 2,69 & 0,00 & 0,00 & 0,00 & 0,00 & 0,00 & 0,00 & 0,00 \\
\hline $67,5^{\circ}$ & 4,25 & 68,84 & 37,37 & 0,00 & 0,00 & 0,00 & 0,00 & 0,00 & 0,00 \\
\hline $90^{\circ}$ Est & 6,88 & 150,71 & 173,90 & 100,49 & $9,79^{\mathrm{E}-13}$ & 0,00 & 0,00 & 0,00 & 0,00 \\
\hline $112,5^{\circ}$ & 8,46 & 209,63 & 290,29 & 260,61 & 167,44 & 46,75 & 0,00 & 0,00 & 0,00 \\
\hline $135^{\circ}$ & 8,75 & 236,64 & 362,47 & 381,05 & 326,81 & 217,15 & 90,49 & 15,17 & 0,00 \\
\hline 157,5 & 7,71 & 227,62 & 379,48 & 443,48 & 436,43 & 366,68 & 251,01 & 134,09 & 34,01 \\
\hline $180^{\circ} \mathrm{Sud}$ & 5,50 & 183,95 & 338,71 & 438,40 & 479,61 & 460,39 & 373,32 & 245,67 & 93,96 \\
\hline $202,5^{\circ}$ & 2,44 & 112,27 & 246,37 & 366,57 & 449,77 & 484,00 & 438,80 & 319,85 & 139,60 \\
\hline $225^{\circ}$ & 0,00 & 26,19 & 116,53 & 238,93 & 351,45 & 433,93 & 437,47 & 345,33 & 163,99 \\
\hline $247,5^{\circ}$ & 0,00 & 0,00 & 6,32 & 74,92 & 199,63 & 317,80 & 369,54 & 318,24 & 163,42 \\
\hline $270^{\circ}$ Ouest & 0,00 & 0,00 & 0,00 & $1,24^{\mathrm{E}-13}$ & 17,42 & 153,28 & 245,35 & 242,70 & 137,96 \\
\hline $292,5^{\circ}$ & 0,00 & 0,00 & 0,00 & 0,00 & 0,00 & 12,18 & 83,80 & 130,21 & 91,50 \\
\hline $315^{\circ}$ & 0,00 & 0,00 & 0,00 & 0,00 & 0,00 & 0 & $1,29^{\mathrm{E}-13}$ & 13,07 & 31,11 \\
\hline $337,5^{\circ}$ & 0,00 & 0,00 & 0,00 & 0,00 & 0,00 & 0,00 & 0,00 & 0,00 & 0,00 \\
\hline$\sum$ & 45,00 & 1218,60 & 1951,47 & 2304,48 & 2428,59 & 2492,21 & 2289,81 & 1764,37 & 855,59 \\
\hline$\sum$ totale & \multicolumn{9}{|c|}{15350,1485} \\
\hline$\%$ & $0,29 \%$ & $7,93 \%$ & $12,71 \%$ & $15,01 \%$ & $15,82 \%$ & $16,23 \%$ & $14,91 \%$ & $11,49 \%$ & $5,57 \%$ \\
\hline
\end{tabular}

\footnotetext{
${ }^{4}$ Ladybug est un composant développé par Sadeghipour Roudsari et al. (2013) pour le plugin de conception paramétrique et de programmation visuelle Dynamo et Grasshopper. Il utilise les moteurs de simulation EnergyPlus (US Department of Energy), RADIANCE (Ward, 1994), et Daysim (Reinhart et Walkenhorst, 2001). Le composant Ladybug utilise le modèle révisé ASHRAE (2009) et Clear Sky (modèle Tau).
} 
Nous constatons alors que, pour $8 \mathrm{~h}, 9 \mathrm{~h}$ et $16 \mathrm{~h}$, le pourcentage de la somme des irradiations solaires directes de toutes les orientations est inférieur à $10 \%$ par rapport à l'irradiation totale. Nous retenons les heures les plus pertinentes de captage d'irradiation directe. Le pourcentage cumulé de l'irradiation de $10 \mathrm{~h}$ à $15 \mathrm{~h}$ est de $86,194 \%$. En se basant sur le principe de Pareto, nous retenons la période de temps qui permet un captage d'au moins $80 \%$ de l'irradiation totale. Ce qui nous amène à fixer le temps de coupure d'ombrage $T_{o}=[10 \mathrm{~h}, 15 \mathrm{~h}]$. Nous constatons que cette durée représente $20 \%$ des $24 \mathrm{~h}$ de la journée.

\subsection{Détermination de la clôture d'ombre à partir du retrait propre en fonction du temps et de l'orientation}

La notion de retrait propre, notée $\mathrm{Rp}$, correspond au recul du bâtiment par rapport aux limites de la parcelle de telle manière que l'ombre soit restreinte à l'intérieur de la parcelle pendant le temps de coupure. Elle sert à définir les limites des clôtures d'ombre (figure 1). Le retrait propre varie en fonction de la date, de l'orientation et de la hauteur de la façade. Il est calculé par la formule suivante (1) :

$$
\mathrm{Rp}=h^{*} \cos \beta / \operatorname{tg} \alpha \quad \text { (mètre) }
$$

Avec :

$h$ la hauteur du bâtiment (en mètre).

$\alpha$ l'angle de la hauteur solaire ${ }^{5}$ (en degré). $\alpha$ exprime le temps.

$\beta$ l'angle entre la normale de la façade et la projection du vecteur solaire au sol (en degré). $\beta$ exprime l'orientation.

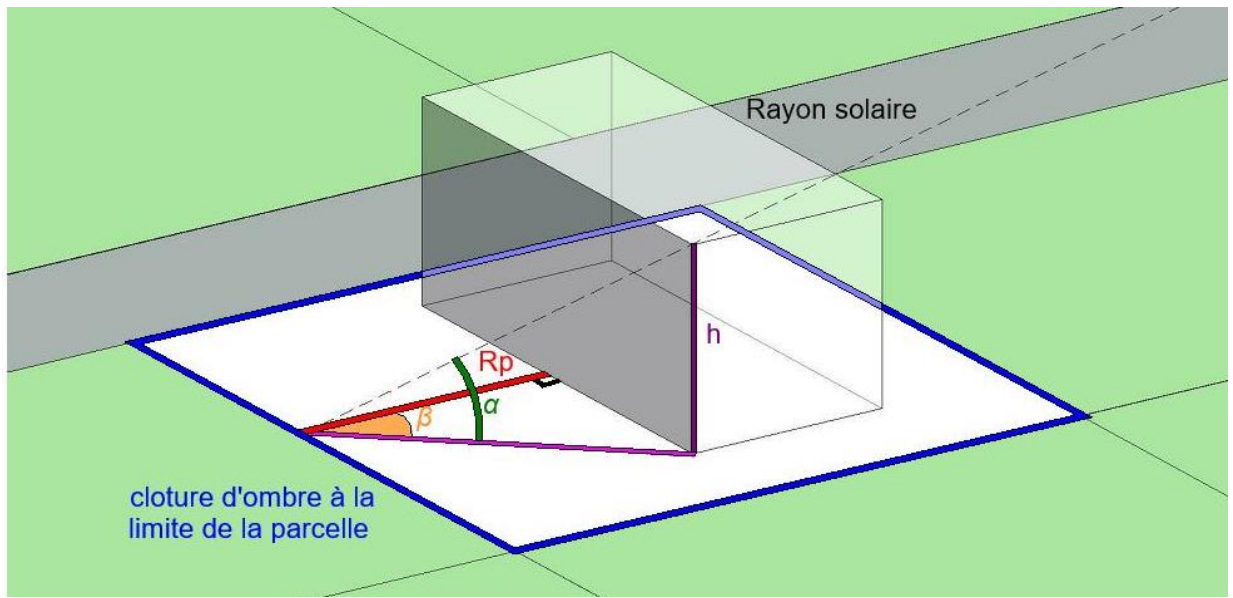

Figure 1. Composantes géométriques du retrait propre

Nous avons développé un composant paramétrique pour le calcul du retrait propre avec l'outil de conception paramétrique Dynamo. Les variables d'entrée sont : l'orientation de la façade, la hauteur de la façade, l'azimut et la hauteur du soleil. La sortie du composant est la valeur du retrait propre (figure 2).

\footnotetext{
${ }^{5}$ La hauteur solaire est l'angle formé par le rayon solaire et le plan horizontal.
} 


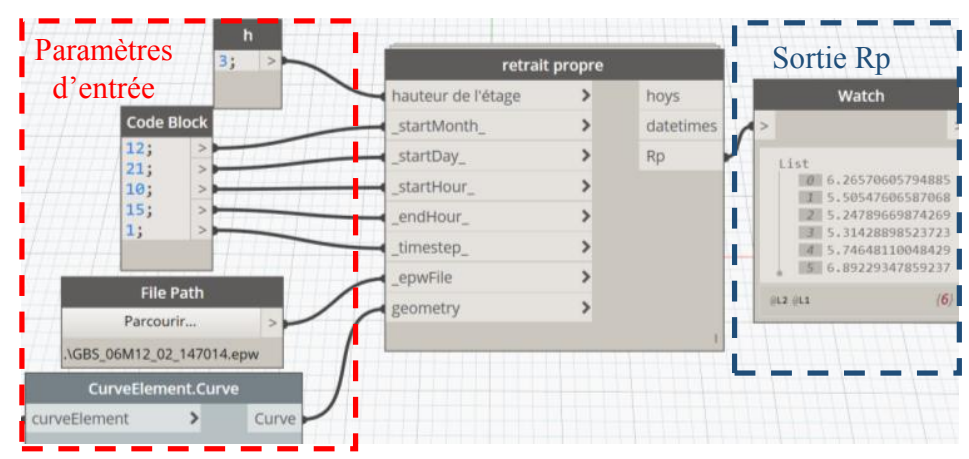

Figure 2. Module de calcul du retrait propre sur Dynamo.

Nous fixons à trois mètres la hauteur d'étage. Nous calculons le retrait propre pour différentes orientations de la façade avec un pas de rotation de $22.5^{\circ}$. La position $0^{\circ}$ indique le Nord. Nous limitons le calcul du retrait propre à la période du temps de coupure [10h, $15 \mathrm{~h}]$ déterminée plus haut. Nous obtenons ainsi seize orientations à étudier. Les valeurs du retrait propre Rp calculées par heure et selon les seize orientations étudiées sont exposées dans le tableau 2.

Tableau 2. Retrait propre $\mathrm{Rp}$ (en mètre) le 21 décembre à Tunis pour seize orientations.

\begin{tabular}{|c|c|c|c|c|c|c|c|c|c|c|}
\hline $\begin{array}{l}\text { Heure } \\
\text { Façade }\end{array}$ & $8 \mathrm{~h}$ & $9 \mathrm{~h}$ & $10 \mathrm{~h}$ & $11 \mathrm{~h}$ & $12 \mathrm{~h}$ & $13 \mathrm{~h}$ & $14 \mathrm{~h}$ & $15 \mathrm{~h}$ & $16 \mathrm{~h}$ & $\begin{array}{l}\mathrm{Rp} \max \\
{[10 \mathrm{~h}, 15 \mathrm{~h}]}\end{array}$ \\
\hline $0^{\circ}$ Nord & 20,48 & 8,39 & 6,27 & 5,51 & 5,25 & 5,32 & 5,75 & 6,91 & 10,73 & 6,91 \\
\hline $22,5^{\circ}$ & 7,30 & 4,46 & 4,19 & 4,33 & 4,69 & 5,32 & 6,39 & 8,55 & 14,87 & 8,55 \\
\hline $45^{\circ}$ & $-6,97$ & $-0,15$ & 1,47 & 2,49 & 3,42 & 4,51 & 6,06 & 8,88 & 16,75 & 8,88 \\
\hline $67,5^{\circ}$ & $-20,20$ & $-4,74$ & $-1,46$ & 0,27 & 1,63 & 3,01 & 4,81 & 7,85 & 16,08 & 7,85 \\
\hline $90^{\circ}$ Est & $-30,35$ & $-8,61$ & $-4,18$ & $-1,98$ & $-0,41$ & 1,06 & 2,82 & 5,64 & 12,96 & 5,64 \\
\hline $112,5^{\circ}$ & $-35,87$ & $-11,17$ & $-6,27$ & $-3,94$ & $-2,38$ & $-1,05$ & 0,40 & 2,56 & 7,87 & 2,56 \\
\hline $135^{\circ}$ & $-35,94$ & $-12,02$ & $-7,39$ & $-5,30$ & $-4,00$ & $-3,01$ & $-2,07$ & $-0,90$ & 1,58 & 0 \\
\hline 157,5 & $-30,53$ & $-11,05$ & $-7,39$ & $-5,85$ & $-5,01$ & $-4,50$ & $-4,23$ & $-4,23$ & $-4,95$ & 0 \\
\hline $180^{\circ}$ & $-20,48$ & $-8,39$ & $-6,27$ & $-5,51$ & $-5,25$ & $-5,32$ & $-5,75$ & $-6,91$ & $-10,7$ & 0 \\
\hline $202,5^{\circ}$ & $-7,30$ & $-4,46$ & $-4,19$ & $-4,33$ & $-4,69$ & $-5,32$ & $-6,39$ & $-8,55$ & $-14,87$ & 0 \\
\hline $225^{\circ}$ & 6,97 & 0,15 & $-1,47$ & $-2,49$ & $-3,42$ & $-4,51$ & $-6,06$ & $-8,88$ & $-16,75$ & 0 \\
\hline $247,5^{\circ}$ & 20,20 & 4,74 & 1,46 & $-0,27$ & $-1,63$ & $-3,01$ & $-4,81$ & $-7,85$ & $-16,08$ & 1,46 \\
\hline $270^{\circ}$ & 30,35 & 8,61 & 4,18 & 1,98 & 0,41 & $-1,06$ & $-2,82$ & $-5,64$ & $-12,96$ & 4,18 \\
\hline $292,5^{\circ}$ & 35,87 & 11,17 & 6,27 & 3,94 & 2,38 & 1,05 & $-0,40$ & $-2,56$ & $-7,87$ & 6,27 \\
\hline $315^{\circ}$ & 35,94 & 12,02 & 7,39 & 5,30 & 4,00 & 3,01 & 2,07 & 0,90 & $-1,58$ & 7,39 \\
\hline $337,5^{\circ}$ & 30,53 & 11,05 & 7,39 & 5,85 & 5,01 & 4,50 & 4,23 & 4,23 & 4,95 & 7,39 \\
\hline
\end{tabular}

Nous remarquons d'après les calculs effectués, que certaines valeurs de $\mathrm{Rp}$ sont négatives. Les ombres projetées sont du côté de l'emprise du bâtiment et non du côté de la parcelle voisine. Il n'y a pas donc de retrait propre pour les valeurs négatives. Nous substituons 0 aux valeurs négatives. 


\section{Stratégies de prospect du droit solaire}

L'étude du retrait propre confirme que la règle de prospect doit tenir compte de l'orientation de la façade. Cependant, la mise en ouvre tel quel de ce constat complique substantiellement la lecture des règlements d'urbanisme. C'est pourquoi nous proposons des stratégies qui intègrent les orientations et les heures de références d'irradiation solaire. Nous développons dans cet article la notion de mutualisation des retraits propres entre deux ou plusieurs bâtiments appartenant à deux ou plusieurs parcelles voisines. Chaque parcelle accepte de recevoir une partie de l'ombre projetée par le bâtiment voisin. Les bâtiments s'implantent alors sur les limites des ombres réciproques.

Nous distinguons deux classes de stratégies de prospect du droit solaire. La première classe ne mutualise pas le retrait propre, définit le prospect par la variation du retrait propre et restreint la projection de l'ombrage aux limites de la parcelle. La deuxième classe définit le prospect par un retrait mutualisé, accepte la projection de l'ombrage au-delà de la limite de la parcelle et se base sur la négociation. Chaque classe comprend trois modalités de retrait: (1) retrait sur-mesure qui calcule le retrait spécifique à chaque orientation de façade, (2) retrait prés-sur-mesure qui calcule le retrait par groupe d'orientations, et (3) retrait commun qui admet un retrait unique garantissant le respect du droit solaire en hiver quelle que soit l'orientation de la façade.

\subsection{Modalités de retrait propre}

\subsubsection{Retrait propre sur-mesure}

Il calcule le retrait propre spécifique à chaque orientation durant les heures du temps de coupure d'ombrage. La valeur du retrait propre retenue est la valeur maximale pour chaque orientation durant le temps de coupure d'ombrage [10h, 15h]. Les valeurs sur-mesure sont indiquées dans le tableau 2 selon l'orientation de la façade.

\subsubsection{Retrait propre prés-sur-mesure}

Nous constatons une corrélation entre la variation des valeurs de retrait propre et celles de l'irradiation directe par groupe d'orientation de façades. Ce constat nous amène à proposer la modalité de retrait prés-sur-mesure regroupant les orientations ayant un même sens de variation des retraits propres et des irradiations directes. Nous obtenons cinq groupes d'orientations avec une variation spécifique de $\mathrm{Rp}$ et de $\mathrm{Rd}$ (figure 3). Nous admettons la valeur maximale de $\mathrm{Rp}$ de chaque groupe garantissant le droit solaire à toutes les façades du groupe. Les groupes d'orientations et les retraits propres retenus sont exposés dans le tableau 3.

Tableau 3. Calcul du retrait propre par groupe d'orientation

\begin{tabular}{|c|c|c|}
\hline Groupe d'orientations & Variation de Rd et Rp & Rp max à [10h, 15h] \\
\hline$\left.] 45^{\circ}, 112.5^{\circ}\right]$ & Rd augmente et Rp diminue & $7,8 \mathrm{~m}$ \\
\hline$\left.] 112.5^{\circ}, 202.5^{\circ}\right]$ & Rd augmente et Rp est nul & $0,0 \mathrm{~m}$ \\
\hline$] 202.5^{\circ}, 247.5^{\circ}[$ & Rd diminue et Rp est nul & $0,0 \mathrm{~m}$ \\
\hline$\left[247.5^{\circ}, 315^{\circ}\right]$ & Rd diminue et Rp augmente & $7,4 \mathrm{~m}$ \\
\hline$\left.] 315^{\circ}, 45^{\circ}\right]$ & Rd est nul et Rp augmente & $8,8 \mathrm{~m}$ \\
\hline
\end{tabular}




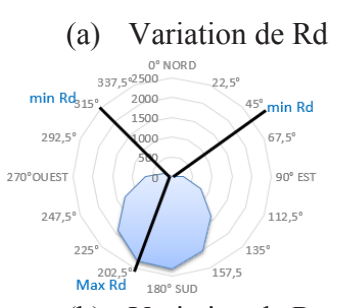

(b) Variation de $\mathrm{Rp}$
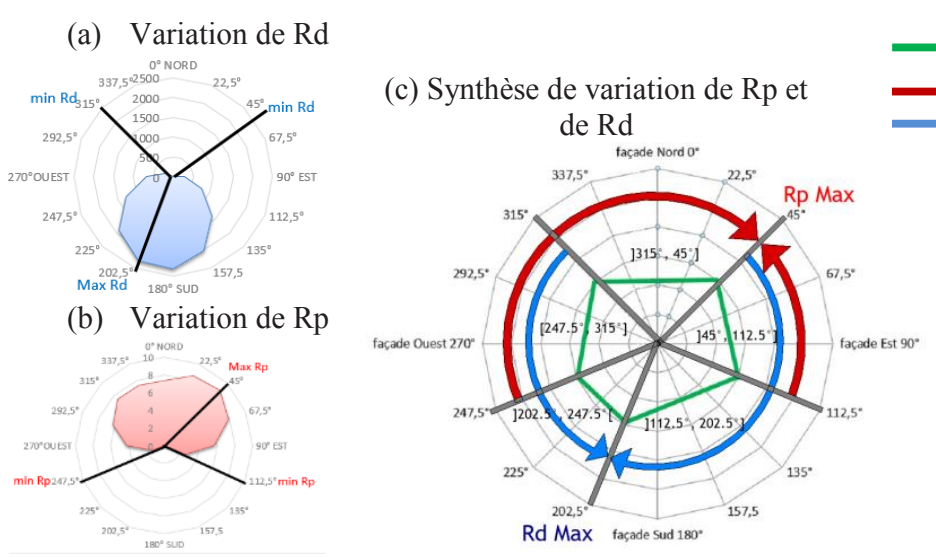

Groupe

d'orientation

Variation $\mathrm{Rp}$

Variation Rd

Figure 3. Groupes d'orientations selon la variation de retrait propre et de l'irradiation directe

\subsubsection{Retrait propre commun}

Il est possible de proposer une modalité de retrait propre commun à toutes les orientations de façades. Il s'agit d'admettre un retrait propre unique garantissant le respect du droit solaire en hiver quelle que soit l'orientation de la façade. Il s'agit du retrait propre maximal pour toutes les heures considérées et toutes les orientations. L'avantage de cette modalité est la simplification de l'application de la règle de prospect. Elle présente en contrepartie l'inconvénient d'être excessive pour certaines orientations. Par exemple, elle fixe la valeur de $\mathrm{Rp}$ à $8.8 \mathrm{~m}$ pour toutes les orientations. Cette valeur est excessive par rapport à la modalité sur-mesure qui attribue un retrait propre de $2.5 \mathrm{~m}$ pour la façade d'orientation de $112.5^{\circ}$.

\subsection{Stratégies du retrait mutualisé}

Nous détaillons ici la classe de stratégie de droit solaire qui définit le prospect par la mutualisation des retraits propres et qui accepte la projection de l'ombrage au-delà de la limite de la parcelle. La mutualisation considère l'environnement urbain dynamique et se base sur la négociation entre les voisins. L'immersion dans une maquette numérique paramétrique offre alors un support interactif de négociation.

\subsubsection{Définition du retrait mutualisé}

Le retrait mutualisé restreint la projection de l'ombrage aux pieds des constructions voisines en se basant sur la négociation.

En partant d'une situation initiale d'implantation des bâtiments selon le retrait propre (figure 4-a), nous distinguons trois cas de mutualisation. Dans le premier cas, nous admettons un retrait mutualisé maximal, noté Rm max, qui est égal à la somme des retraits propres des bâtiments A et B. Ce qui permet d'augmenter la hauteur des bâtiments A et B (figure 4-b). Dans le deuxième cas, nous admettons un retrait mutualisé minimal, noté Rm min, qui est égal à la valeur maximale des deux retraits propres des deux bâtiments. Ce qui permet d'augmenter la hauteur pour le bâtiment ayant un retrait propre minimal (figure 4c). Pour le troisième cas, nous admettons une valeur comprise entre le retrait mutualisé minimal et le retrait mutualisé maximal. Ce qui permet d'augmenter la hauteur des bâtiments A et B (figure 4-d) selon l'accord des deux voisins. 
Ces trois cas de détermination du retrait mutualisé (figure 4) permettent une négociation de la hauteur des constructions et de l'implémentation des bâtiments par rapport à la limite séparative. Dans le cas d'un retrait propre nul, exemple de la façade sud, les trois cas cidessus se réduisent à une seule variante.

L'implantation des deux bâtiments selon la distance Rm qui les sépare est un objet de négociation. L'implantation peut être d'une manière symétrique par rapport à la limite séparative de la parcelle ou asymétrique selon le critère de négociation et l'accord entre voisins.

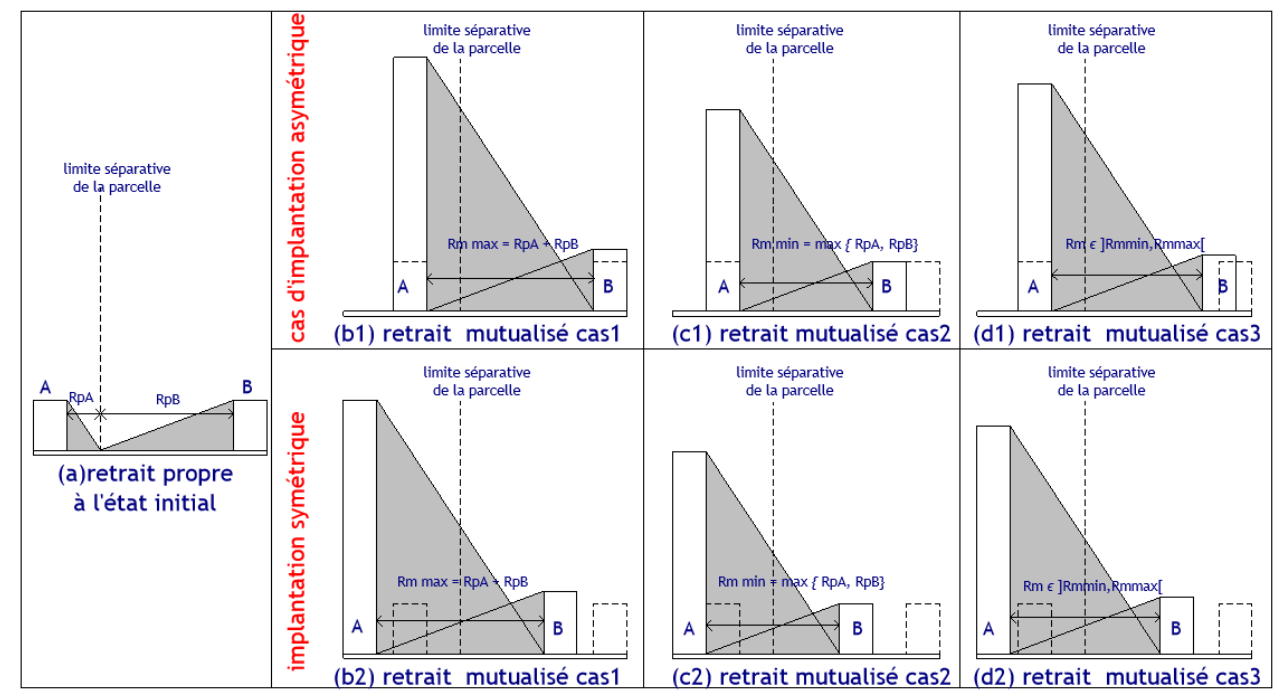

Figure 4. Stratégies de calcul du retrait mutualisé entre deux parcelles A et B. (variante d'implantation symétrique et variante d'implantation asymétrique)

\subsubsection{Application de la stratégie de retrait mutualisé}

Nous avons explicité ci-dessus trois modalités de retrait: sur-mesure, prés-sur-mesure et commun. Le cas le plus précis est la modalité de retrait mutualisé sur-mesure qui tient compte de l'orientation de chaque façade. Pour cette modalité, le retrait mutualisé et la hauteur fluctuent selon l'orientation de la façade. La modalité de retrait mutualisé prés-surmesure propose une mesure de retrait mutualisé par groupe d'orientations. Pour cette modalité, le retrait mutualisé et la hauteur fluctuent selon le groupe d'orientation. La modalité de retrait mutualisé commun admet une mesure unique du retrait mutualisé et de la hauteur.

Nous développons dans ce qui suit (tableau 4) le cas d'un retrait mutualisé sur-mesure pour le cas d'implantation symétrique par rapport à la limite séparative. Nous considérons l'exemple de deux façades $\mathrm{A}$ et $\mathrm{B}$ orientées respectivement à $247.5^{\circ}$ et $67.5^{\circ}$. Le retrait propre de la façade A est de $1.4 \mathrm{~m}$ et celui de la façade B est de $7.8 \mathrm{~m}$. Trois cas se présentent alors : (1) $\mathrm{Rm} \max =9.2 \mathrm{~m}$ et il est possible d'augmenter la hauteur de A et $\mathrm{B},(2)$ le retrait minimal est de $7.8 \mathrm{~m}$ et il est possible d'augmenter la hauteur pour la façade dont le $\mathrm{Rp}<7.8 \mathrm{~m}$, (3) choisir une valeur intermédiaire de $\mathrm{Rp}$ dans l'intervalle ]7.8m, $9.2 \mathrm{~m}$ [ et il est possible d'augmenter la hauteur de A et B. 
Tableau 4. Illustration du retrait propre mutualisé avec implantation symétrique après mutualisation.

Cas de façades orientées $67.5^{\circ}$ et $247.5^{\circ}$ pendant le temps de coupure [10h, 15h].

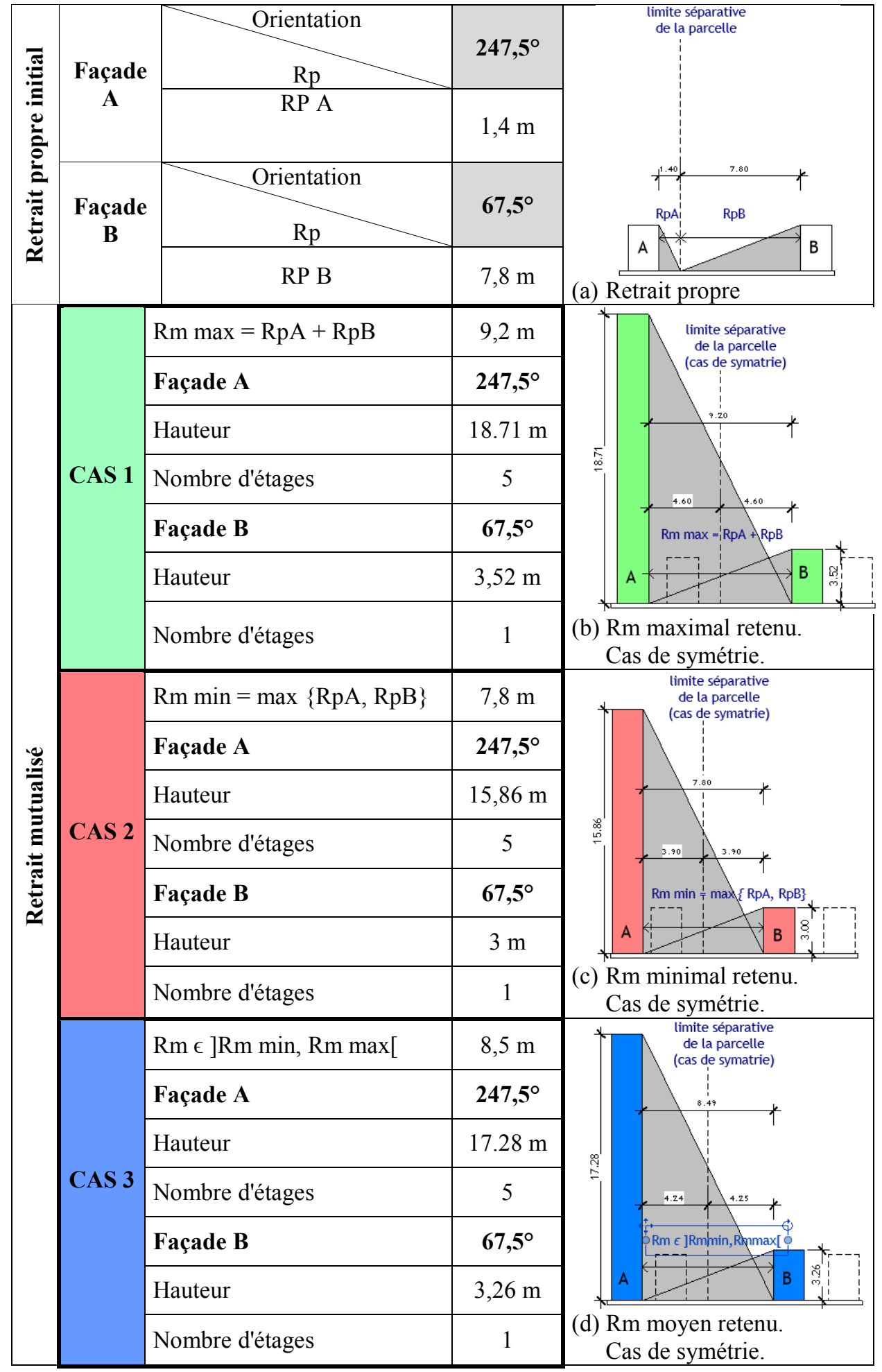




\section{Discussion}

Chaque cas de négociation et de mutualisation traduit une vision du processus de densification. Dans le cas de retrait mutualisé maximal, nous avons des valeurs maximales de retraits et de hauteurs qui traduisent une densification verticale. Dans le cas de retrait mutualisé minimal, nous avons des valeurs minimales de retraits et de hauteurs qui traduisent une densification horizontale. Dans le troisième cas, plus la valeur choisie de l'intervalle s'approche du retrait mutualisé maximal, plus la solution tend vers une densification verticale, et plus elle s'approche du retrait mutualisé minimal, plus elle tend vers une densification horizontale. Nous avons donc ici, en plus du modèle de contrôle solaire, un instrument de planification urbaine.

Ce travail se limite à étudier les différentes stratégies et modalités qui préservent le droit solaire en milieu urbain. Cependant, dans un pays où les mois chauds durent plus que les mois froids, il est important d'étudier des stratégies et des modalités pour préserver le droit d'ombrage. Cette notion a été proposée par Raboudi (2017). Le droit d'ombrage définit les conditions d'ombrage entre bâtiments. Il désigne l'aptitude d'une construction à projeter son ombre pour protéger les bâtiments voisins du rayonnement solaire direct indésirable en été. Raboudi (2017) propose l'enveloppe d'ombrage comme dispositif géométrique permettant de préserver le droit d'ombrage. Nous proposons en continuité de ce travail d'étudier l'application des stratégies et des modalités de prospect pour l'ombrage. Il serait aussi intéressant d'utiliser les techniques d'optimisation multi objectifs pour trouver un optimum global entre les stratégies et les modalités d'accès solaire en hiver et celles d'ombrage en été.

Dans cet article, nous avons étudié seulement l'aspect énergétique de l'accès solaire. Il serait aussi important d'inclure d'autres aspects tels que les apports solaires diffus et les inter-réflexions, d'étudier d'autres facteurs tels que l'éclairage et le confort physique. D'autre part, nous avons calculé l'irradiation solaire au solstice d'hiver. Une étude plus étalée sur les mois les plus froids permettrait d'optimiser le potentiel de captage énergétique et de donner des résultats plus fins pour délimiter le temps de coupure d'ombrage. Il serait également important de déterminer le temps de coupure d'ombrage selon la consommation énergétique des constructions (chauffage et climatisation). Par exemple, dans l'hypothèse d'installation de dispositifs de captage énergétique, si l'intervalle du temps de coupure permet d'atteindre des valeurs d'énergie qui excèdent les besoins d'une construction, le temps de coupure pourrait être réduit au profit du voisin.

La notion de retrait mutualisé pourrait intégrer d'autres critères d'optimisation, tels que la densification urbaine, les besoins énergétiques des bâtiments et le confort physique des occupants.

\section{Conclusion}

Cet article présente un modèle de stratégies de prospect dotées de modalités préservant le droit solaire. Il propose la notion de retrait propre et de retrait mutualisé. La stratégie présentée sert à l'établissement d'une réglementation urbaine interactive respectant le contexte géo-climatique et intégrant l'avis des acteurs (voisins). Nous pouvons alors passer d'un cahier des charges statiques à un modèle paramétrique dynamique et immersif via une maquette numérique. Ce qui permet une plus grande flexibilité et interactivité. Les résultats de cette recherche s'appliquent aussi bien pour la conception de bâtiments dans de nouveaux tissus urbains que pour l'intervention dans des anciens tissus. Ils peuvent également fournir une base pour définir des orientations pertinentes pour la génération de parcellaires et de nouveaux tracés urbains. 


\section{Bibliographie}

American Society of Heating, Refrigerating and Air-Conditioning Engineers. (2009) ASHRAE Handbook: Fundamentals. Volume 10 de 2009 Ashrae Handbook Fundamentals. ASHRAE Handbook Fundamentals Systems-International Metric System. Belkaid, A. (2014). Morphose du volume englobant réglementaire. Université de Carthage. Chabbi, M. (2004). L'urbanisation en Tunisie, transformations et tendances d'évolution. Dans Villes réelles villes projetées, villes maghrébines en fabrication. Paris : Maisonneuve et Larose - Paris.

De Luca, F. (2017). From Envelope to Layout. Buildings Massing and Layout Generation for Solar Access in Urban. ShoCK Sharing Computational Knowledge. In Proceedings of the 35th eCAADe Conference - Volume 2. pp. 431-440. Rome.

Harzallah, A. (2007). Emergence et évolution des préconisations solaires dans les théories architecturales et urbaines en France, de la seconde moitié du XIXème siècle à la deuxième guerre mondiale. Université de Nantes.

Hobday RA., \& Dancer SJ. (2013). Roles of sunlight and natural ventilation for controlling infection: historical and curret perspectives, Journal of Hospital Infection 2013. http://dx.doi.org/10.1016/j.jhin.2013.04.011

Knowles, R. (1981). Sun Rhythm Form. Cambridge, Massachusetts and London, England, MIT Press, 1981.

Pareto, V. (1965) «Essai sur la courbe de la répartition de la richesses », dans Recueil publié par la faculté de droit à l'occasion de l'exposition nationale suisse, Genève, Université de Lausanne, 1896 ; réédité dans Vilfredo Pareto, Écrits sur la courbe de répartition de la richesses, Genève, Droz, 1965

Plan d'aménagement urbain de la commune de Tunis, (2016).

Raboudi, K., Belkaïd, A., \& Ben Saci, A. (2011). Système multi agents pour la modélisation du volume englobant solaire. In Proceedings of the Biskra Architecture and Sustainability International Conference (BASC'2011). Biskra.

Raboudi, K., \& Ben Saci, A. (2014). Génération de volumes de contrôle solaire. In J.-C. J.C. Bignon, G. Halin, \& S. Kubicki (Eds.), Interaction(s) des maquettes numériques : Acte du 6ème Séminaire de Conception Architecturale Numérique (SCAN'14) (pp. 221-231). Luxembourg: Presses universitaires de Nancy.

Raboudi, K. (2017). Générateur morphologique de règles urbaines de contrôle solaire. Université de Carthage.

Reinhart, C. F., Walkenhorst, O., 2001, "Dynamic RADIANCE-based daylight simulations for a full-scale test office with outer venetian blinds." Energy \& Buildings, 33:7 pp. 683697.

Sadeghipour Roudsari, M., Pak, M., \& Smith, A. (2013). Ladybug: a Parametric Environmental Plugin for Grasshopper To Help Designers Create an EnvironmentallyConscious Design. In Proceedings of 13th Conference of International Building Performance Simulation Association, 3129-3135.

Siret, D. (1997). Propositions pour une approche déclarative des ambiances dans le projet architectural : Application à l'ensoleillement. Université de Nantes.

Vartholomaios, A. (2015) 'The residential solar block envelope: A method for enabling the development of compact urban blocks with high passive solar potential', Energy and Buildings. Elsevier B.V., 99, pp. 303-312. doi: 10.1016/j.enbuild.2015.04.046.

Ward, G.J. 1994. The RADIANCE Lighting Simulation and Rendering System. In Proceedings of the 21st Annual Conference on Computer graphics and interactive techniques, Orlando. 